\title{
Why small-scale testing of reagents goes wrong
}

\author{
PD Fawell CSIRO Mineral Resources Flagship, Australia \\ AD Costine CSIRO Mineral Resources Flagship, Australia
}

AF Grabsch CSIRO Mineral Resources Flagship, Australia

\begin{abstract}
Small-scale cylinder or jar tests are routinely applied to establish the impact of polymer reagents that induce aggregation in mineral systems. Despite their value, such tests are often done poorly or under too narrow a range of conditions, which can lead to trends being either missed or wrongly interpreted. A number of common errors are discussed, together with strategies to maximise the practical value of the results obtained.
\end{abstract}

\section{$1 \quad$ Introduction}

The use of high molecular weight water-soluble polymers (flocculants) to induce aggregation is well established within tailings thickening, and addition of similar reagents to high solids suspensions for additional dewatering is now considered both viable and advantageous in some tailings deposition applications. Small-scale batch testing of reagents is common in application development and optimisation; these are typically in stirred tanks/beakers or in measuring cylinders. Pilot or full-scale continuous trials are clearly preferable but are not always practical, even when an operating site is available. Feed properties (solids concentration, particle size, mineralogy) can be variable, making the comparison of reagents problematic, and sometimes there is limited scope to run larger scale trials of sufficient duration to obtain reliable, stable trends. In the case of greenfields developments, small-scale testing is unavoidable, particularly for initial reagent selection.

A frequently expressed concern is that small-scale testing of reagents can give results that are either irreproducible or inconsistent with full-scale performance. The latter manifests as (for example) reagents not giving the expected enhanced performance, much higher dosages being required or the inability to achieve a targeted settling flux. Irreproducibility can usually be put down to issues with the procedures applied, whereas any mismatch between small and full-scale trends can reflect a wide range of issues. These can include major shortcomings in the way reagents are dosed that prevent anywhere near their full performance being realised. While such shortcomings are a serious concern, they are (at least in the case of thickening) considered in detail elsewhere (e.g. Nguyen et al. 2012) and will not be discussed here.

This paper evaluates the many mistakes that can be made in the small-scale testing of high molecular weight water-soluble polymers that are used to assist in sedimentation and dewatering processes in mineral applications, and provides recommendations as to how they can be avoided or their impact minimised. The examples used mainly come from the study of feedwell applications, but consideration is also given to reagent dosing to high solids suspensions.

\section{Errors in procedure}

\subsection{Preparation for testing}

Even before considering procedures for testing the performance of a reagent, there are several aspects of the starting materials that need to be understood to ensure maximum test reproducibility. These relate to both the feed slurry and the solution of the reagent to be dosed. 


\subsubsection{What is the particle size of the feed?}

While the size distribution for the solids within a feed slurry is a critical factor in determining a system's response to reagent addition, many studies fail to provide proper size characterisation. Sieve information will often produce a lower fraction that is $-45 \mu \mathrm{m}$ (i.e. 'fines'), but this fraction hides the key detail on the feed that determines both aggregation and dewatering responses. There will be distinctly different performance from fines that are predominantly in the 1-10 $\mu \mathrm{m}$ range relative to those from 10-30 $\mu \mathrm{m}$. Much more valuable characterisation is obtained from a full particle size distribution (PSD) measured by laser diffraction/scattering instruments, particularly when they offer access to sizes below $1 \mu \mathrm{m}$.

However, it is dangerous to assume such PSDs tell the full story. The laser diffraction/scattering response is volume-weighted, and therefore the broad PSDs seen with many tailings will be dominated by larger solids. This may reflect particle mass contributions well, but aggregation responses to reagents are determined to a much greater degree by particle number. Relatively minor peaks in the fines region can in fact outnumber the coarse phases by many orders of magnitude. Broad, multimodal PSDs can therefore be difficult to correlate with flocculation performance, even leading to predictions that run counter to observations.

Instruments that can reliably provide particle count information are not common. Automated counting can be achieved by allowing particles and aggregates to pass singly through an electrical sensing zone, their presence then detected by a suitable sensor (Gregory 2009). Such pulse counting is highly accurate and the Coulter counter is perhaps the best known instrument of this type. However, it requires very high solids dilution when applied to mineral suspensions, making accuracy dependent upon sampling. In addition, the lower size limit of around 1-2 $\mu \mathrm{m}$ may not be appropriate for some clay feeds. Chord length distributions from focused beam reflectance measurement (FBRM) extend to just below $1 \mu \mathrm{m}$ and can be acquired in real time without the need for solids dilution, but while particle number sensitive, the technique does not give number counts. Heath et al. (2002) found that the non-linear response of FBRM to solids concentration limits its ability to detect changes in particle number. The G series FBRM probes (released 2012) appear to offer much greater sensitivity to 1-10 $\mu \mathrm{m}$ particles and a more linear response to solids concentration, increasing the potential for real-time monitoring of feed particle properties (as applied in the next Section).

In the absence of access to particle count information, the value of laser diffraction/scattering techniques can be enhanced by sieving feeds to remove the coarser solids $+45 \mu \mathrm{m}$ and then measuring the PSD of the fine fraction, knowing its mass contribution to the full sample. It is also advisable to measure PSDs both with and without dispersion (the former involving sonication and perhaps dispersant addition) to understand the level of pre-existing aggregation.

The feed itself must be suited to the desired task. For example, Rey (1988) sought to examine the impact of solution cations on flocculant activity, but chose a coal tailings slurry that was coarse $(50 \%<19 \mu \mathrm{m}$, only $10 \%<3 \mu \mathrm{m}$ ), varied from $4-6 \mathrm{wt} / \mathrm{vol} \%$ and contained a mixture of phases, all factors making it less than ideal for identifying potentially subtle variations in flocculant solutions.

\subsubsection{Handling the feed slurry}

Clays are a common component of almost all tailings slurries and are well known to hinder dewatering performance, particularly when montmorillonite is a significant component. This is largely a consequence of the plate-like, small clay particles and their loose packing when uncontrolled dispersion takes place in liquors with low electrolyte concentrations (de Kretser et al. 1997). Calcium addition to such slurries favours dewatering by inducing face-face coagulation of the platelets, thereby reducing particle packing volumes, but this is not always a practical option at the scale of many tailings operations.

Another consequence of a higher clay content is that feed slurries are rarely at a high state of dispersion, and can therefore be highly shear sensitive. The potential impact of this is demonstrated within Figure 1(a), in which an FBRM probe is used to monitor the total counts in a synthetic clay-quartz slurry stirred at a fixed rate in a baffled container. The counts were found to more than double over a 20-hour period, and it can be expected that the actual particle number increase would be even higher. While the rate of change 
was highest over the first four hours, the release of particles still continued well after this and showed no sign of abating. Only minor changes would be seen in the corresponding volume-based distributions.
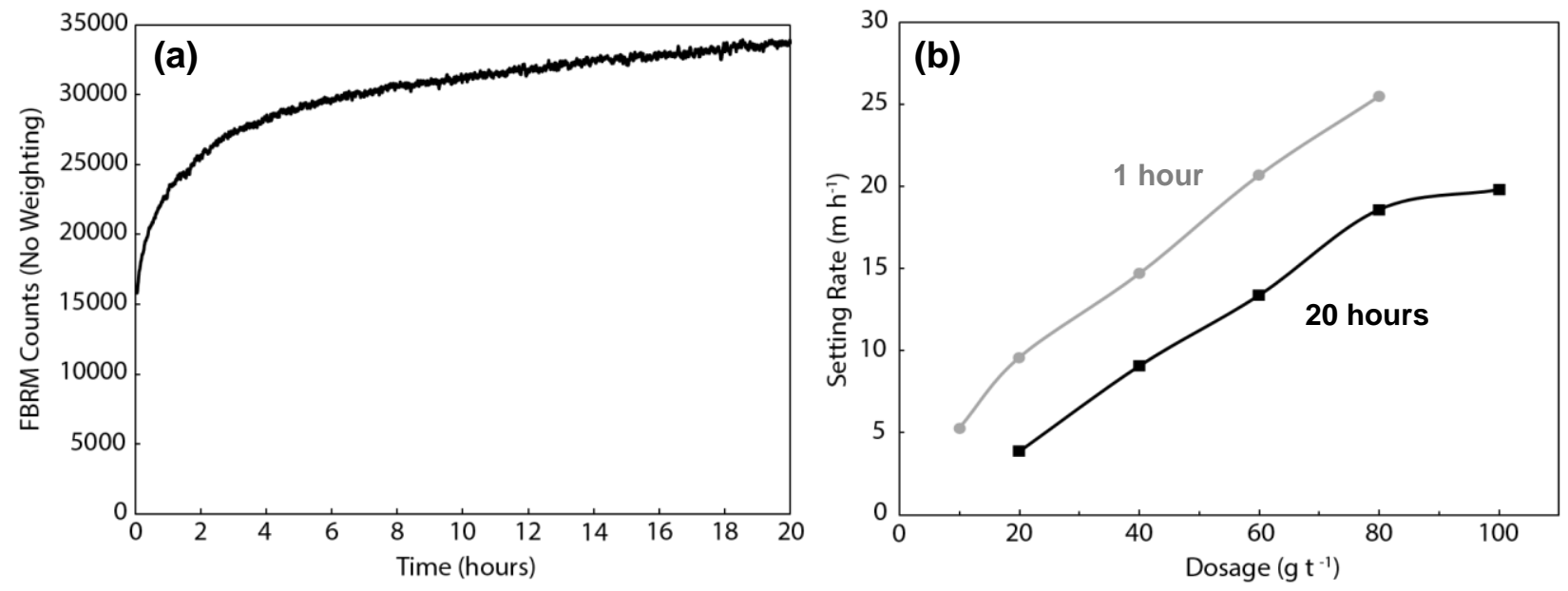

Figure 1 (a) unweighted FBRM (G400) counts as a function of time for a $100 \mathrm{~kg} \mathrm{~m}^{-3} \mathrm{mix}$ of clay and quartz stirred at $800 \mathrm{rpm}$; (b) dosage response curves for the flocculation of samples taken at one and 20 hours (diluted to $40 \mathrm{~kg} \mathrm{~m}^{-3}$ ) with BASF Magnafloc 336 (0.01 wt\%)

Comparison of the settling rate response to flocculant dosage for samples taken at one and 20 hours clearly indicates a shift to higher dosages after extended mixing (Figure 1(b)), with double the dosage required to achieve a settling rate of $10 \mathrm{~m} \mathrm{~h}^{-1}$. Also of interest is evidence of a plateau in settling rate at higher dosages for the 20-hour sample, reflecting a lower optimum solids concentration as a consequence of the extra release of fines.

In the context of small-scale testwork, such shifts in dosage response over the course of a series of measurements make useful comparisons highly problematic. When the potential for shear sensitivity of a feed is suspected, it is advisable to initially condition the slurry at a high mixing intensity and then reduce to the minimum intensity to provide slurry homogeneity during testing. If testing is paused, ceasing slurry mixing altogether should also be considered.

\subsubsection{Handling reagents}

The time-dependent nature of high molecular weight polymer solutions used as flocculants is now well studied (e.g. Owen et al. 2002; 2007), and while issues can still be expected to be encountered with the make-up and handing of such solutions at full-scale operations, there should be no excuses with laboratory testing. The main concerns are with powder products, for which make-up becomes more difficult to achieve reproducibly as the concentration increases. Concentrations of $1 \mathrm{wt} \%$ are often quoted, but for higher molecular weight products, $0.5 \mathrm{wt} \%$ is a more practical upper limit. For small-scale testing, suitable ageing times of stock flocculant solutions prior to use can generally be provided, with 18-24 hours sufficient to ensure activity is both close to optimal and not rapidly changing. While stock solutions may maintain high activity for many weeks, it is good practice to not use them after a week, especially if being compared against other products.

The advantage of emulsion-based products is that they give high activity almost immediately upon make-up, but it is a mistake to assume this is optimal activity. Owen et al. (2007) showed that high molecular weight emulsion products still required close to the same time as powders to disentangle after make-up to then offer maximum flocculation performance. With emulsions requiring intense mixing for inversion during make-up, there is also the danger of over-shearing that leads to a permanent reduction of activity. If examining an emulsion product for the first time, several tests to optimise make-up are advisable, given that the conditions being applied may not necessarily match those recommended by the supplier. 


\section{$2.2 \quad$ Test procedures}

The waste water industry has long applied 'jar tests', in which a reagent is dosed into a beaker or tank containing slurry with an impellor used to provide agitation for a fixed duration. However, such tests are intended for much lower solids concentration applications and primarily for aggregation induced with coagulants. The residence times under shear are hence longer and the sensitivity to shear is far less than in higher solids tailings feedwell applications. The situation is more complex in the context of high solids/high dosage tailings applications, although sensitivity to the duration of applied shear is still a major issue.

Cylinder tests are more common in testing for feedwell applications, with mixing of slurry and reagent then achieved either by cylinder inversion or plunger strokes. The primary advantage of the cylinder geometry is the scope for the accurate measurement of mudline settling as a guide to flocculation performance. Cylinder mixing is also easier to control than in stirred beakers. Much is often made of wall effects within cylinders, and different cylinder sizes will inevitably produce different settling rates, but Farrow and Swift (1996) clearly demonstrated this reflected distinct mixing regimes - by flocculating externally and filling cylinders of varying diameter, the initial settling rates were all identical. However, wall effects are significant on consolidation (Lester et al. 2014), and therefore final settled volumes will be affected.

Some common procedural errors are discussed below, and where practical, these are demonstrated through examples from the published literature.

\subsubsection{Dosed concentration of reagent}

Coagulant solution properties are easier to control than those of high molecular weight polymers. As discussed in Section 2.1.3, stock solutions of the latter are quite viscous, and inadequate dilution prior to dosing does not favour the polymer's mixing and distribution through the slurry and often results in high dosages. The reagent solution will always represent a minor volume in relation to the slurry. Efficient distribution through the slurry is critical, as adsorption for most feeds at practical dosages is rapid. Dilution of the dosed solution aids distribution by increasing its volume while reducing viscosity. The latter is critical, as viscous solutions have been shown to mix with difficulty even in turbulent pipe flow (Mohanarangam et al. 2010). The impact of flocculant dilution on aggregation is illustrated in Figure 2(a), which shows aggregate size as monitored by FBRM as a function of reaction time under turbulent pipe flow. The slower mixing of the more concentrated flocculant delays the onset of aggregation and limits the size that can be attained. The counts $<10 \mu \mathrm{m}$ (Figure 2(b)) reflects fines capture, which is also poor in the absence of high dilution.
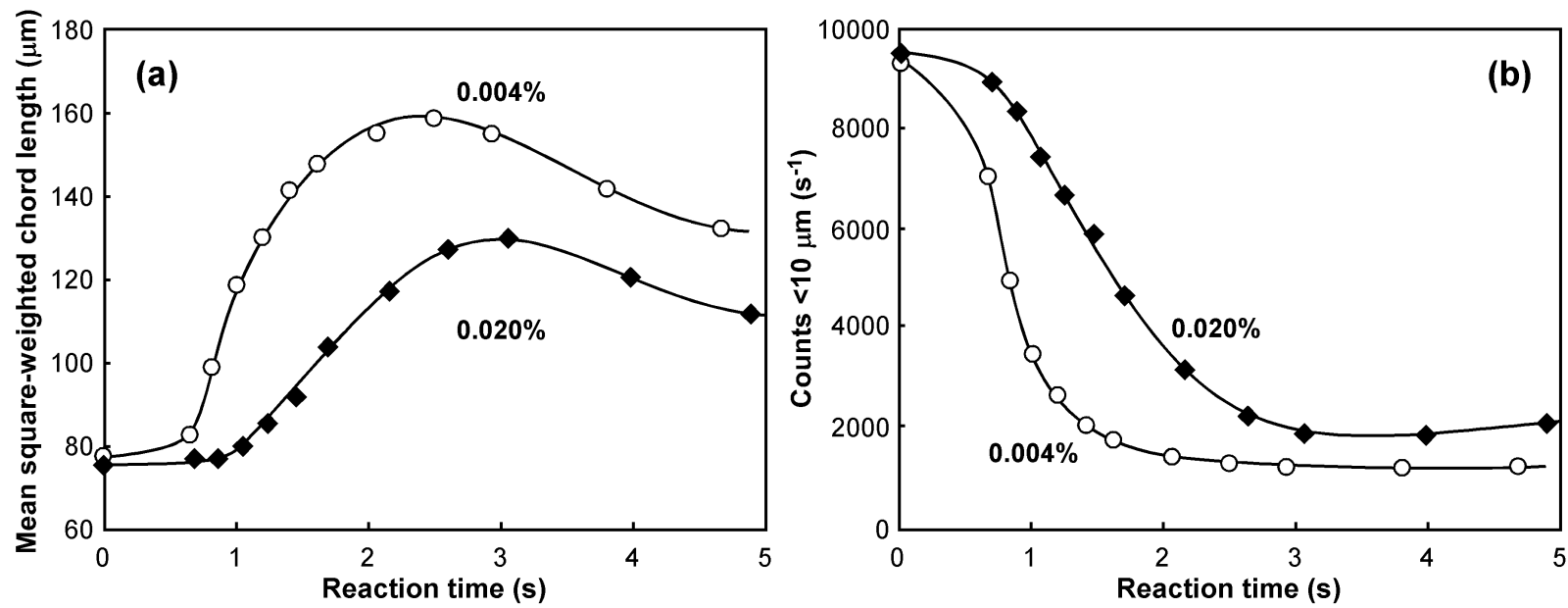

Figure 2 Effect of flocculant concentration on FBRM (M500) statistics for flocculation of an industrial tailings feed

( $90 \mathrm{~kg} \mathrm{~m}^{-3}$, dosage $\left.25 \mathrm{~g} \mathrm{t}^{-1}\right)$ in a $22 \mathrm{~mm}$ ID linear pipe reactor $\left(25 \mathrm{~L} \mathrm{~min}^{-1}\right)$, showing (a) mean square-weighted chord length and (b) unweighted counts $<10 \mu \mathrm{m}$ as a function of time 
Examples of inadequate flocculant dilution can be seen in the work of Mpofu and co-workers (Mpofu et al. 2003; 2004), who applied flocculants at $0.1 \mathrm{wt} \%$ to clay slurries under fairly mild mixing and at high dosages. The mild mixing may have been more appropriate at a lower flocculant concentration, possibly reducing the required dosages. A diluted flocculant concentration of $0.02 \%$ would be far more suitable, with further dilution to $0.01 \%$ potentially better. In low solids applications or when supernatant clarity is a priority, even further dilution can be an option.

At the elevated dosages required when adding reagents into high solids concentration feeds, the scope for reagent dilution is much more limited in the full-scale application. However, in most small-scale testing, the objective is to better understand the process, and therefore it should be a priority to establish whether a product's superior performance is a consequence of changed aggregation physics (kinetics or aggregate structure) or simply a reflection of a lower viscosity.

\subsubsection{Comparisons at a fixed dosage}

There are numerous examples within the literature where the performance of different flocculant products are compared at a single fixed dosage (e.g. Caskey \& Primus 1986; McFarlane et al. 2008), and this is a common approach in many site-based product evaluations. Such comparisons may be appropriate when flocculation is only a preparative stage to evaluate properties further downstream (i.e. rheology, filtration, deposition), but it is difficult to envisage when this can be justified for detailed studies of the flocculation process. Dosage response curves are never linear, and their shapes will often vary for different flocculant products, which means trends observed at one dosage may change at higher or lower dosages, particularly for distinct molecular weights and chemistries. Few products will have the same molecular weight, so comparisons purely on a grams per tonne basis are only relevant when product cost is the driving issue. Single dosage comparisons also suffer as products will have different viscosities, which impacts on aggregation performance (as discussed previously in this paper) unless done at high dilution of reagent solutions.

It is therefore always preferable to acquire full dosage response curves, covering dosages from the onset of aggregation through to (where possible) high settling rates well above what may be required. In some cases, this may only represent a narrow working range of dosages, and it is entirely likely that the working range for different products may not overlap, particularly when comparing emulsion and powder products (comparisons are best done on the basis of active polymer contents, but rarely are in practice).

\subsubsection{Excessive settling rates}

Few operating tailings thickeners would need to seek settling rates much greater than $\sim 30 \mathrm{~m} \mathrm{~h}^{-1}$. Very few experimentalists can reliably measure settling rates of $\sim 40 \mathrm{~m} \mathrm{~h}^{-1}$ by eye, given that would represent a mud-line falling at $11 \mathrm{~mm} \mathrm{~s}^{-1}$, assuming the mud-line is distinct (often not the case at high settling rates). While it is possible to capture these settling rates by recording images of the settling mud-line, with the system described by Zhu et al. (2000) being just one example, at such large aggregate sizes the sensitivity to small variations in dosage and mixing conditions will often cause substantial shifts, making the values obtained highly questionable for comparisons.

Despite this, there are cases where settling rates over $100 \mathrm{~m} \mathrm{~h}^{-1}$ are quoted and values over $40 \mathrm{~m} \mathrm{~h}^{-1}$ are contrasted. Figure 3 shows results from two studies that illustrate this problem. Chen (1998) conducted settling tests with a kaolin suspension to compare the performance of six cationic flocculants. The dosage response curves (Figure 3(a)) suggest two of the products gave settling rates in excess of $100 \mathrm{~m} \mathrm{~h}^{-1}$, with one approaching $300 \mathrm{~m} \mathrm{~h}^{-1}$. However, the dosages are high and the majority of the points appear to fall on a plateau in the settling response; whether each plateau is real is questionable, given that the errors involved in each point must be large. The region of greatest interest for the dosage response falls under $500 \mathrm{~g} \mathrm{t}^{-1}$, in which there is only one point for each flocculant. As it turns out, the range of behaviour for the products was so large that the interpretation of relative behaviour is still likely to be correct. 

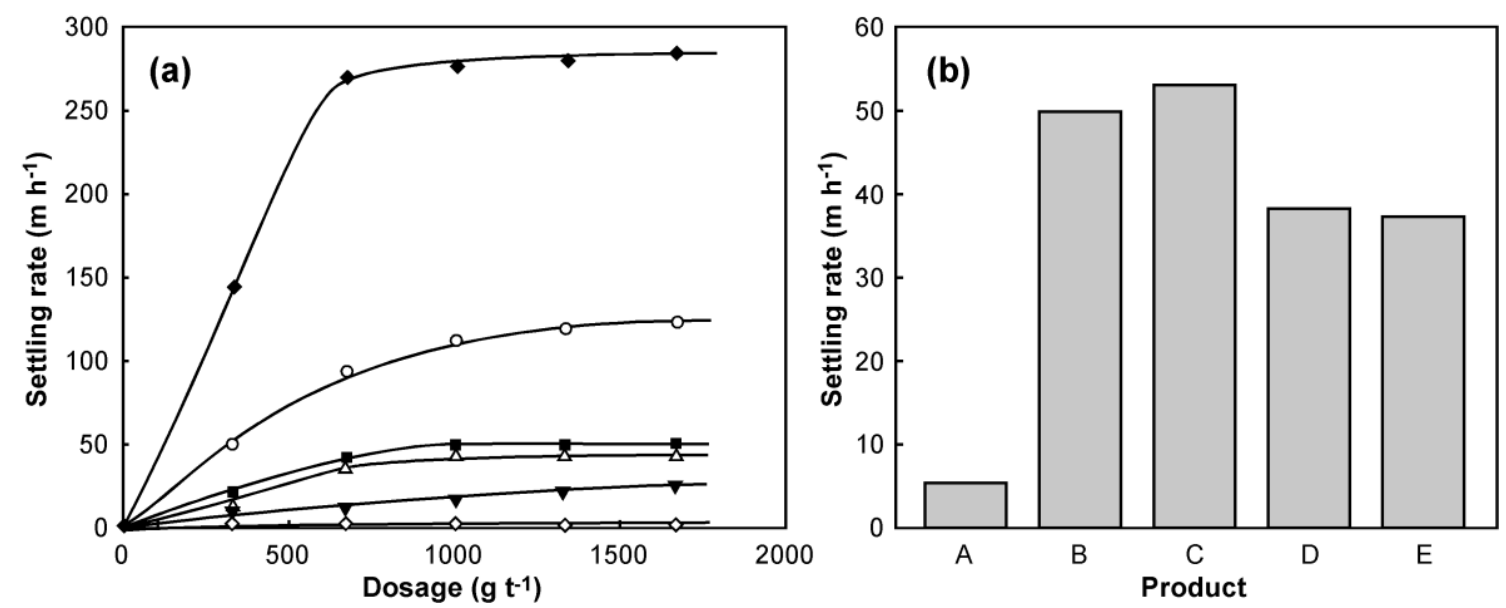

Figure 3 (a) dosage response for six cationic flocculants when applied to a $30 \mathrm{~kg} \mathrm{~m}^{-3}$ kaolin suspension (adapted from Chen 1998); and (b) comparison of cylinder setting rates for five flocculants applied at $50 \mathrm{~g} \mathrm{t}^{-1}$ to a $10 \mathrm{wt} \%$ quartz suspension (unpublished results)

This is not the case for the results in Figure 3(b) comparing quartz flocculation by five flocculants of similar molecular weight at one dosage. The inferior performance of product $A$ is apparent, but no conclusions can be drawn on the relative performance of the others. With settling rates all near $40-50 \mathrm{~m} \mathrm{~h}^{-1}$, the differences are too small when the potential for errors and varying dosage responses are considered. Once again, full dosage response curves are required over a settling rate range that spans practical operating conditions.

\subsubsection{Comparisons at a fixed solids concentration}

Fixing on a single solids concentration is a very common practice in testing and can be acceptable if done after a number of concentrations have already been screened. However, a fixed concentration that is too high or low raises concerns, especially when different flocculants are being compared. At too high a concentration, the sensitivity to differences between products may be reduced and even masked by mixing effects (Dollimore \& Horridge 1973; Besra et al. 2002; Sabah \& Cengiz 2004).

If one product produces a denser aggregate than another, then the relative response may also vary with solids concentration. Grabsch et al. (2013) compared two different flocculant chemistries over a range of solids concentrations (Figure 4) to confirm that one was favoured at higher solids concentrations. 


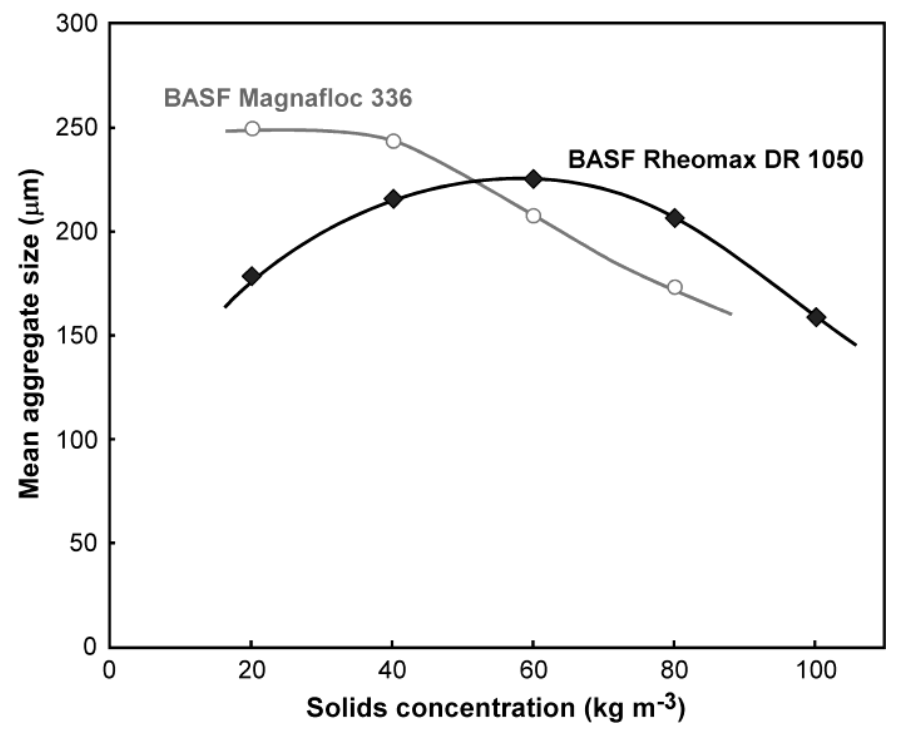

Figure 4 Mean aggregate sizes for calcite flocculation in turbulent pipe flow (7.7 $\mathrm{mm}$ ID, reaction time $4.9 \mathrm{~s}$, mean shear rate $\left.1,270 \mathrm{~s}^{-1}\right)$ with Magnafloc ${ }^{\circledR} 336$ and Rheomax $^{\circledR}$ DR 1050 (BASF) at $80 \mathrm{~g} \mathrm{t}^{-1}$ (adapted from Grabsch et al. 2013)

The practical significance of this difference is potentially quite large, with 3D computational fluid dynamics (CFD) modelling of feedwell flows based on these results indicating throughput could double when operating with higher solids feeds, reducing the need for solids dilution prior to flocculation (Tanguay et al. 2014). Even when considering one product, it should be recognised that the solids concentrations of feeds being delivered to a feedwell are often highly variable, and so flocculant dosage response curves should ideally always be measured at a range of solids levels.

With flocculation usually sensitive to solids concentration, the best test procedures involve a known volume of slurry being added to a cylinder with an appropriate diluant and the required flocculant dose then making-up to the full volume. In this way, dosage can be varied while maintaining solids. However, many studies involve simply adding variable flocculant volumes to the slurry (e.g. Mpofu et al. 2004). When considering a range of dosages, small changes in solids concentration can have an impact; the use of high flocculant concentrations may negate that effect, but as already discussed, adds another complexity.

\subsubsection{Poorly defined/excessive mixing}

Many groups apply 'jar test' procedures from water treatment science to thickening-related studies (Ofori et al. 2011; Sabah \& Erkan 2006), although such an approach is arguably highly inappropriate. Reaction times in stirred tanks of several minutes are appropriate for low solids clarification by coagulation, but rarely for flocculation at higher solids concentrations. Distinct zones of shear will exist in a stirred tank, with the highest shear near the impellor (Figure 5). For coagulation at low solids, the higher shear is required to provide the necessary collision rate, so most of the 'action' takes place in this zone. When used for flocculation, aggregation will take place in all zones, with the high shear zone likely to contribute to both aggregate growth and breakage. Reproducibility is difficult at short reaction times, but at longer times the 'bulk' flocculation response will end up as a blend from the different shear regimes, often over-sheared and therefore inferior to what could be achieved from a narrower shear rate distribution over a shorter time. The consequences may be the selection of higher dosages than are actually required and the masking (or even reversal) of performance trends. 


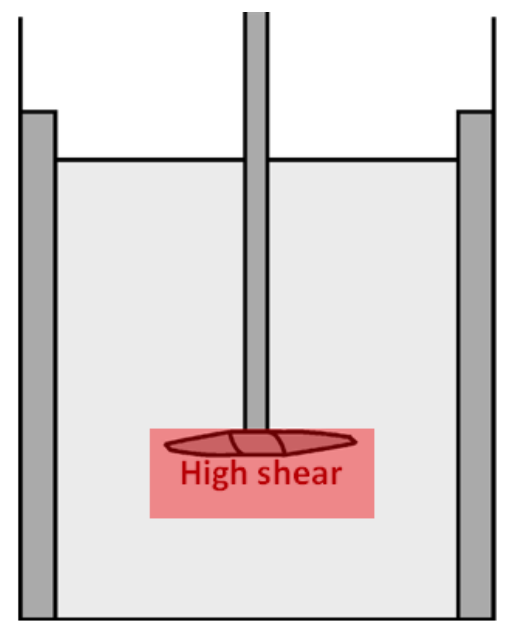

(a)

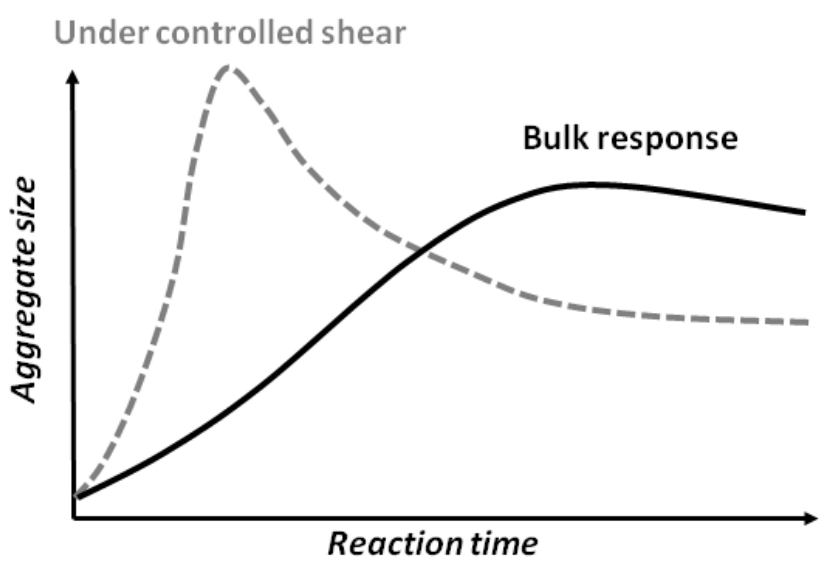

(b)

Figure 5 Schematic representations of (a) high shear zone near an impellor in stirred tank mixing; and (b) the corresponding bulk reaction profile for aggregation, also indicating what the response may be under a more controlled (narrow) shear regime

Mixing is hardly well defined in cylinders, but the variation of plunger strokes or cylinder inversions does provide a degree of control over the duration of mixing that is more appropriate for studies related to feedwell or launder/feedpipe flocculation. The challenge is to ensure the reproducibility of such mixing. Automated cylinder rotators have been used (e.g. Scott et al. 1996), as have piston-driven plungers (e.g. Jones et al. 1998), but for fully manual tests the best strategy is to ensure the same operator conducts all the tests in a program. Note that cylinder tests are unlikely to be suitable for the mixing of reagents in high solids applications, with stirred tank tests at least then offering the option of continuous testing.

\subsubsection{Assuming different products require the same mixing}

The level of mixing required to induce aggregation will vary not only with solids concentration and flocculant solution concentration, but also with flocculant selection. A low molecular weight product that produces more bridges can be expected to tolerate more mixing during flocculation, which can favour fines capture, but higher molecular weight products may be far more sensitive to such mixing. The impact of this is demonstrated in the results obtained by Clark et al. (1990), who used a standard kaolinite slurry to quantify the effects of both molecular weight and anionic charge density on settling rates (Figure 6). While their study produced numerous valuable insights, their error was to apply 20 cylinder inversions in the testing of each product, a protocol identified in earlier work with lower molecular weight cationic flocculants. This was likely to be excessive for anionic flocculants, and certainly for the higher molecular weight products. Over-shearing during flocculation would contribute to the plateaus observed in the dosage response (Figure 6(a)). 

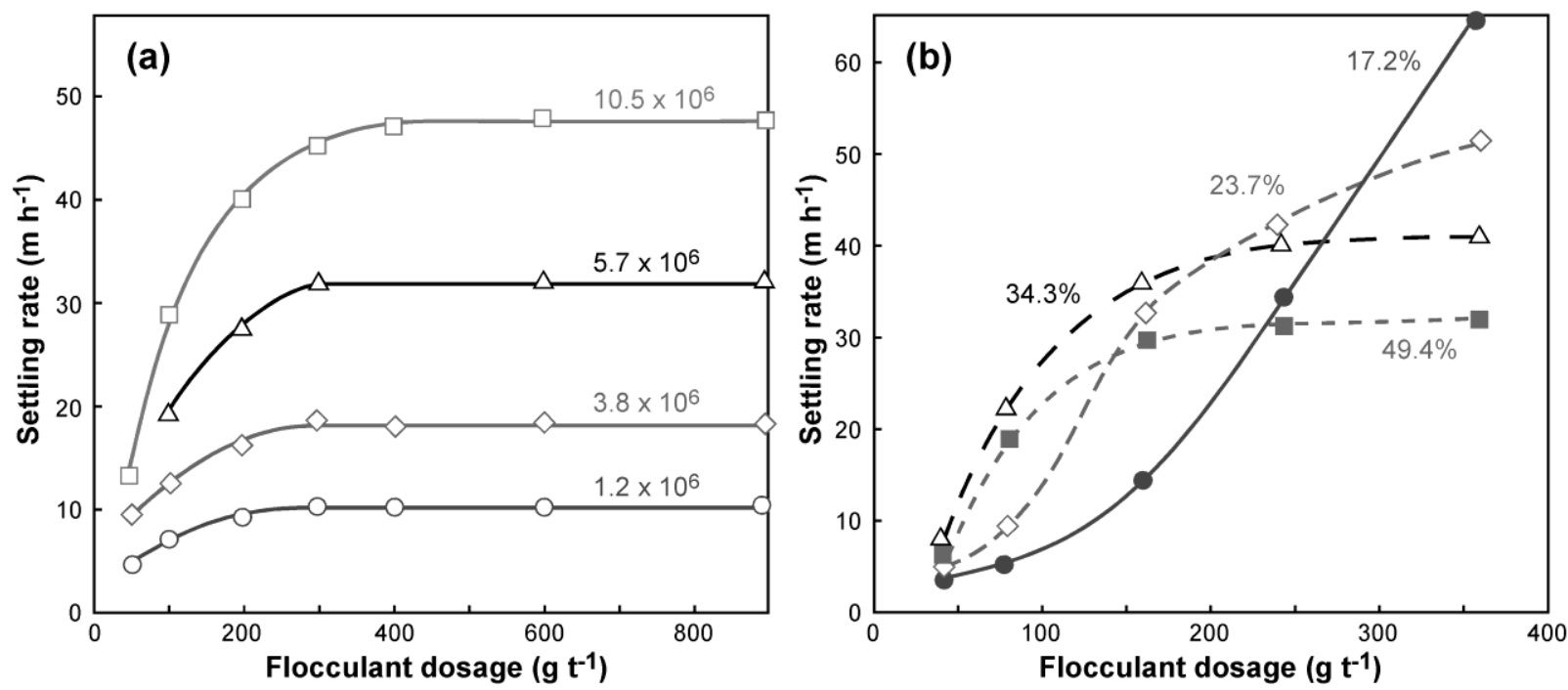

Figure 6 Settling rate versus dosage after addition to $20 \mathrm{~kg} \mathrm{~m}^{-3}$ kaolinite and 20 cylinder inversions for (a) $21-24 \%$ anionic flocculants of varying mean molecular weight; and (b) 13-15 $\times 10^{6}$ molecular weight flocculants of varying anionic charge (adapted from Clark et al. 1990)

That plateaus were more evident for products of higher charge densities (Figure 6(b)) may be evidence of a change in aggregate density and strength, such that solids concentration effects play some role in limiting settling rates - with only one shear regime considered, this cannot be confirmed from these results. Optimising the mixing for each product may not have been a practical option, but to have at least considered a lower shear regime (i.e. fewer cylinder inversions) would have provided a much better indication of the relative behaviour of the products and the true impacts of molecular weight and charge density.

\section{$3 \quad$ Errors in interpretation}

While there is clearly a need to ensure the reproducibility of small-scale test procedures, this alone is insufficient, as any results and conclusions obtained will still only be relevant to the specific conditions of that test. The danger then comes from (i) the use of conditions that either miss or mask key aspects of the actual potential performance, or (ii) not considering the specific operational requirements for a process when designing or interpreting experiments.

An example of the former can be seen in studies into how flocculant solutions are affected by inorganic cations that influence to varying degrees the conformation of acrylamide/acrylate copolymers. Such effects are expected to be reflected in the flocculation activity of the polymer solutions, but literature in this area is far from convincing, particularly on the influence of divalent cations. Henderson and Wheatley (1987) claimed the presence of calcium and magnesium ions in flocculant solutions did not influence subsequent clay flocculation, arguing the 1:1 complexes with carboxylate functionalities had a relatively minor effect on flocculant solution conformation. However, Rey (1988) observed a significant loss of activity with tailings from flocculant dilution in liquor with increasing calcium concentrations; a similar effect from calcium was attributed by Peng and Di (1994) to polymer coiling and precipitation on the clay surface.

Witham et al. (2012) resolved this conflict by using continuous flocculation of a standard substrate in a Couette mixing device (rotating inner bob/rotor, stationary outer cylinder) allowing operator-independent settling rate measurements to be made on the output slurry, with much greater control achieved over both the residence time under shear and its intensity. Figure 7 shows the impact of three different applied shear regimes during flocculation with an anionic product that was diluted to its working concentration in a liquor containing either sodium, magnesium or calcium ions. At the lowest shear (Couette rotation rate $300 \mathrm{rpm}$ ) the flocculant diluted in the presence of divalent cations could still readily form moderate size aggregates 
with setting rates up to $10 \mathrm{~m} \mathrm{~h}^{-1}$, but it was only at higher dosages that the flocculant's reduced coil dimensions had an impact, making it much harder to form larger, fast settling aggregates. Larger aggregates are intrinsically weaker, and so this behaviour is only seen under the milder applied shear. In contrast, the highest shear regime studied $(600 \mathrm{rpm})$ led to aggregate breakage being dominant at higher dosages and thereby masking the influence of cations on the flocculant's apparent activity. The same effect would be seen from prolonged residence times under shear during the flocculation process.
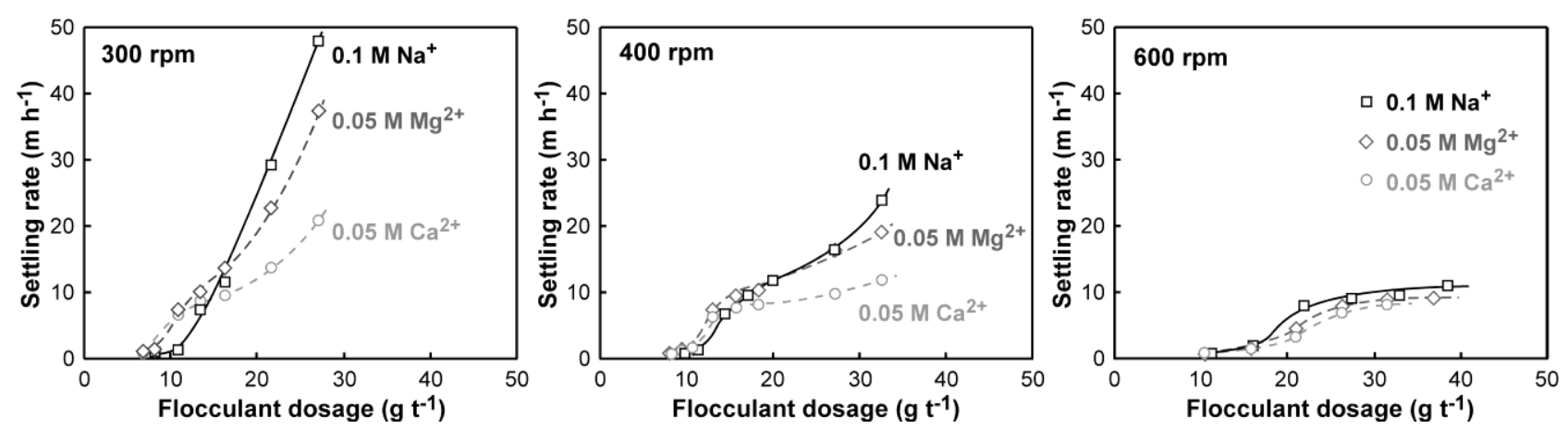

\section{Figure 7 Settling rate as a function of flocculant dosage at three different rotation rates on continuous flow through a Couette mixing device for an anionic flocculant diluted to 0.005 wt\% in $0.1 \mathrm{M} \mathrm{Na}^{+}, 0.05 \mathrm{M} \mathrm{Mg}^{2+}$ or $0.05 \mathrm{M} \mathrm{Ca}^{2+}$ (adapted from Witham et al. 2012)}

While the Couette mixing device made the study of such effects simpler, the same trends could just as easily have been identified through the variation of cylinder inversions or plunger strokes. These results also highlight another important consideration, in that practical interpretation as to whether they play a significant factor in feedwell and thickener performance will very much depend on the unit in question. For example, if in conventional thickeners for which the nominal liquor rise rate is low (e.g. $<5 \mathrm{~m} \mathrm{~h}^{-1}$ ), then it is quite likely that settling rates comfortably in excess of that may still be achieved with many substrates even with flocculants affected by the presence of cations. Liquor rise rates will increase at higher throughputs, with high values also a feature of more modern high rate or paste thickeners; the ability to achieve high settling rates then becomes critical, and under these conditions the quality of the liquor used to dilute flocculants would definitely become a major issue.

Relevant examples of where flocculation testwork is related to full-scale operational performance are almost impossible to find, and while they are much more common in vendor or consultant reports, such data is not available for publication. However, the following general observations can be made:

- Dosage curves for different high molecular weight products may be used to highlight the advantage of one product over the remainder through its ability to give high settling rates at the lowest dosage, and this is valid when high settling rates are required. However, when the nominal liquor rise rate is low, then there may not be a need for high molecular weights. Lower molecular weight products can in fact give moderate settling rates with some substrates that exceed those from a higher molecular weight at the same dosage, simply as a consequence of providing more chains for bridging. This can also be a consideration in feedwell systems known to be under-sheared.

- Small-scale testing often seeks to identify and apply optimum conditions for aggregation, and yet the full-scale applications of the process are frequently poorly optimised. Proper quantification of the likely fluid flows within a feedwell is best obtained from CFD (e.g. Nguyen et al. 2012), but in many cases it is possible to conclude whether a feedwell is under or over-sheared. The former will typically see much of the liquor surface within the feedwell quiescent, with little evidence of a developed swirling flow; for the latter, the entire surface will appear highly turbulent. Testing regimes should at least take such observations into account; for example, the best product selected under optimal mixing conditions may lead to weaker aggregates and therefore require 
much higher dosages in a high shear environment. In this case it would be advisable to also test the impact of higher shear simply by applying additional cylinder inversions or plunger strokes.

- It is the fines (particles $<20 \mu \mathrm{m}$ ) within a feed that dominate aggregation processes. Many tailings feed streams will contain a broad range of particle sizes, and there is clearly a preference to conduct small-scale testing on 'real' feeds. Certainly solids up to 100 $\mu \mathrm{m}$ in size have strong potential to be aggregated with fines and thereby accelerate the measured settling rates. However, larger particles in the order of $300-400 \mu \mathrm{m}$ or larger are much less likely to be captured in aggregates. In small-scale testing they will rapidly segregate and possibly disrupt the aggregate formation process; of greater concern is that such solids are much harder to reproducibly sample in slurry splitting, which has the potential to influence solids concentration determinations. If this coarse phase is a relatively minor fraction (say $10-20 \%$ of the total mass), it may be better to remove them by sieving prior to testing and then subsequently correct the derived solids concentration.

- There is a tendency to screen many reagents over a very limited range of experimental conditions (often only at one set of conditions), and yet the majority of commercial products that are available offer variations in charge density or molecular weight that may only give incremental performance changes, i.e. products offering variations in functional chemistry are comparatively few. In seeking step-changes in performance, it is far better to first screen a smaller number of carefully selected products across a broader range of conditions (solids concentration, applied shear), and if necessary then chose conditions for more specific product property optimisation.

\section{$4 \quad$ Other small-scale tests}

\subsection{Polymer addition at thickening solids concentrations}

While jar and cylinder tests remain the simplest options with the potential to be done at the smallest scale, there are other approaches that offer the potential for screening without going to pilot-scale. As alluded to above, flocculation during continuous flow through a Couette mixing device as developed by Farrow and Swift (1996) provides the ability to rapidly screen a wide range of conditions and products with high reproducibility. The earliest version had a rotor diameter of $200 \mathrm{~mm}$ and a gap width of $5 \mathrm{~mm}$, but later versions scaled this down to a rotor of $100 \mathrm{~mm}$ diameter (Owen et al. 2002, 2007; Witham et al. 2012), thereby reducing required flow rates, providing easier access to milder mixing conditions and far greater portability. At this reduced size, $\sim 20 \mathrm{~L}$ of slurry could provide $\sim 60-70$ minutes of operation, and with FBRM detection proving useful to confirm stability at any set of conditions, full dosage curves for several products could easily be acquired.

Others have applied similar designs in flocculation studies (e.g. Gagnon et al. 2002). Rulyov et al. (2006) produced an 'ultraflocculator' with a rotor diameter of only $30 \mathrm{~mm}$ and gap of $2 \mathrm{~mm}$, through which the flow rate was typically just $3.5 \mathrm{~L} \mathrm{~min}^{-1}$. Excellent flocculation was claimed from the application of a short burst of very high shear, although the relevance of this to practical feedwell flocculation is questionable.

Couette devices provide efficient and highly reproducible mixing conditions, but are not fully turbulent under the conditions of interest and therefore it is not a simple process to extract a mean shear rate. Options for controlling the residence time under shear are also limited, making it difficult to examine flocculation kinetics. Turbulent pipe flow with FBRM detection allows reaction times to be controlled to a fraction of a second and the ready estimation of mean shear rates, although the initial version with a linear $22 \mathrm{~mm} \mathrm{ID} \mathrm{pipe} \mathrm{required} \mathrm{slurry} \mathrm{flow} \mathrm{rates} \mathrm{of} \mathrm{at} \mathrm{least} 20 \mathrm{~L} \mathrm{~min}^{-1}$ (Heath et al. 2006). Grabsch et al. (2013) scaled this down to an ID of $7.7 \mathrm{~mm}$ with lengths $0.30-22.0 \mathrm{~m}$, coiled to a diameter of $0.3 \mathrm{~m}$, greatly reducing the experimental footprint and required flow rates $\left(1-3 \mathrm{~L} \mathrm{~min}^{-1}\right)$. Secondary flow effects are well known within helical coils, affecting the calculated shear rates and raising the Reynolds numbers required for turbulence, but in this case the coil is too large and the flow velocities too low for this to be significant. 
Carissimi and Rubio (2015) applied $12.5 \mathrm{~mm}$ ID flexible tubing of $12 \mathrm{~m}$ length and coiled to a diameter of $0.1 \mathrm{~m}$ in coagulating and flocculating a range of substrates, generally at low solids concentrations.

The main concern with such scaled-down reactors is maintaining turbulence, which means that some of the lower mean shear rates of interest cannot be examined. Caution also needs to be taken to limit the size of aggregates formed to minimise any potential for settling during transit through the reactors. Within these limitations, small-scale turbulent pipe flow is ideal for quantification of the kinetics of aggregate growth and breakage when both aggregate size and settling rate can be measured on the outflow (Grabsch et al. 2013). With only settling tests being performed, this is still an improved approach for isolating the impacts of applied shear intensity and duration. Critically, it is also ideal as a preparative tool; for example, Gladman et al. (2010) used a $12.5 \mathrm{~mm}$ ID pipe of lengths 1.8 and $9.66 \mathrm{~m}$ (residence times 2 and $10 \mathrm{~s}$, respectively, at $6.3 \mathrm{~L} \mathrm{~min}^{-1}$ ) to produce flocculated calcite feed for the examination of consolidation in a $5 \mathrm{~m}$ raked column.

\subsection{Polymer addition to high solids suspensions}

Post-thickener polymer addition at elevated dosages has great potential to speed water recycle and is actively being trialled or applied at some operations across the minerals and petroleum industries. The issues addressed in the preceding sections, however, are further heightened in high solids tailings applications, and as a result many of the fundamental aspects of the process remain poorly understood. A major weakness of most small-scale studies in this area is the almost inevitable poor control over the properties of the initially thickened solids, due to poor mixing and dispersion of the viscous polymer solutions in the dense tailings suspensions. Yao et al. (2012) and Mizani et al. (2013) treated mature fine tailings at $\sim 35 \mathrm{wt} \%$ solids with $0.4 \%$ polymer solutions in impellor-stirred vessels, with the latter study dosing 'near the impellor' for short reaction times (10 s). The wide shear rate distribution encountered in such vessels can result in higher consumption of polymer, overdosing and significant variations in the treated tailings characteristics for duplicate tests. If the issues associated with optimisation of mixing can be resolved, there is then scope to properly identify reagent properties (e.g. molecular weight and charge density) for optimum dewatering performance.

One promising alternative to the turbulent dispersion of viscous polymer solutions is chaotic advection, as the governing mixing principles are independent of fluid rheology and flow rate, and so mixing is expected to be robust across a wide range of processing conditions (Lester et al. 2009). Given that the aggregate structures formed in this process are already exposed to intense breakage events (due to the high number of colliding particles), generating chaotic laminar flows may be a preferred approach. The stretching and folding mechanism of chaotic advection creates material striations whose thickness decay exponentially with time, making it possible to design fast and efficient mixing systems which operate at low shear or small dimensions. This class of mixer shows distinct promise because mixing is not driven by the dynamics of the flow field itself, but rather through the underlying geometry of the mixing device.

A small-scale chaotic mixer was used to continuously mix $30 \mathrm{wt} \%$ kaolin slurries and $0.4 \%$ polymer solutions via a slow braiding motion of a series of vertical rods $(28 \times 0.6 \mathrm{~cm})$, with a G400 FBRM probe providing real time monitoring of aggregate sizes (Costine et al. 2014). The mixer consisted of three fixed rods rotating at $35 \mathrm{rpm}$ and three planetary rods which traced out a cycloid pattern around the fixed rods at $52 \mathrm{rpm}$. This low shear dynamic mixer applied a uniform shear history to the entire slurry, allowing for the controlled formation of well-defined, robust aggregate structures with favourable dewatering characteristics.

\section{$5 \quad$ Conclusions}

The most important message to be drawn from the above discussion is that the simple tests routinely applied to test polymer reagents are anything but simple, with the very real prospect for product comparisons to be biased by the selected test conditions. There is no single recommended procedure that fits all applications, with the better option instead being to first carefully consider the objectives of the test and select conditions that maximise both the relevance of the results and their sensitivity to the main variables. Tests that are to be related to full-scale operations should be done within any operational 
constraints, e.g. high concentration of the reagent to be dosed, although always remaining conscious of the potential impacts on the results. More fundamental studies should be free of such constraints, and yet many published studies fail to properly isolate the interactions of the polymer reagents with a solid substrate from the physical properties of reagent solutions, substrate concentration or the applied shear regime.

\section{Acknowledgement}

Some portions of the work within this paper were either conducted within the AMIRA P266 'Improving Thickener Technology' series of projects or involved techniques derived from those projects. The ongoing support of the companies that support AMIRA P266 is gratefully acknowledged.

\section{References}

Besra, L, Sengupta, DK, Roy, SK \& Ay, P 2002, 'Studies on flocculation and dewatering of kaolin suspensions by anionic polyacrylamide flocculant in the presence of some surfactants', International Journal of Mineral Processing, vol. 66, pp. 1-28.

Carissimi, E \& Rubio, J 2015, 'Polymer-bridging flocculation performance using turbulent pipe flow', Minerals Engineering, vol. 70, pp. 20-25.

Caskey, JA \& Primus, RJ 1986, 'The effect of anionic polyacrylamide molecular-conformation and configuration on flocculation effectiveness', Environmental Progress, vol. 5, pp. 98-103.

Chen, WJ 1998, 'Effects of surface charge and shear during orthokinetic flocculation on the adsorption and sedimentation of kaolin suspensions in polyelectrolyte solutions', Separation Science and Technology, vol. 33, pp. 569-590.

Clark, AQ, Herrington, TM \& Petzold, JC 1990, 'The flocculation of kaolin suspensions with anionic polyacrylamides of varying molar mass and anionic character', Colloids and Surfaces, vol. 44, pp. 247-261.

Costine, A, Lester, D, Fawell, P \& Chryss, A 2014, 'Shear isn't mixing: how to build larger aggregates using chaotic advection for accelerated dewatering', in RJ Jewell, AB Fourie, PS Wells \& D van Zyl (eds), Proceedings of the 17th international seminar on paste and thickened tailings, InfoMine, Vancouver, paper no. 49.

de Kretser, R, Scales, PJ \& Boger, DV 1997, 'Improving clay-based tailings disposal: Case study on coal tailings', AIChE Journal, vol. 43, pp. 1894-1903.

Dollimore, D \& Horridge, TA 1973, 'Dependence of flocculation behavior on China clay-polyacrylamide suspensions on suspension $\mathrm{pH}^{\prime}$, Journal of Colloid and Interface Science, vol. 42, pp. 581-588.

Farrow, JB \& Swift, JD 1996, 'A new procedure for assessing the performance of flocculants', International Journal of Mineral Processing, vol. 46, pp. 263-275.

Gagnon, MJ, Simard, G, Charette, A \& Péloquin, G 2002, 'Characterization of red mud aggregate populations generated under shear conditions', CIM Bulletin, vol. 95, no. 1062, pp. 87-91.

Gladman, BR, Rudman, M \& Scales, PJ 2010, 'Experimental validation of a 1-D continuous thickening model using a pilot column', Chemical Engineering Science, vol. 65, pp. 3937-3946.

Grabsch, AF, Fawell, PD, Adkins, SJ \& Beveridge, A 2013, 'How achieving a higher aggregate density through flocculant selection can impact upon polymer-bridging flocculation', International Journal of Mineral Processing, vol. 124, pp. 83-94.

Gregory, J 2009, 'Monitoring particle aggregation processes', Advances in Colloid and Interface Science, vol. 147-48, pp. 109-123.

Heath, AR, Fawell, PD, Bahri, PA \& Swift, JD 2002, 'Estimating average particle size by focused beam reflectance measurement (FBRM)', Particle \& Particle Systems Characterization, vol. 19, pp. 84-95.

Heath, AR, Bahri, PA, Fawell, PD \& Farrow, JB 2006, 'Polymer flocculation of calcite: Experimental results from turbulent pipe flow', AlChE Journal, vol. 52, pp. 1284-1293.

Henderson, JM \& Wheatley, AD 1987, 'Factors effecting a loss of flocculation activity of polyacrylamide solutions: shear degradation, cation complexation, and solution ageing', Journal of Applied Polymer Science, vol. 33, pp. 669-684.

Jones, F, Farrow, JB \& van Bronswijk, W 1998, 'Flocculation of hematite in synthetic Bayer liquors', Colloids and Surfaces A Physicochemical and Engineering Aspects, vol. 135, pp. 183-192.

Lester, DR, Rudman, M \& Metcalfe, G 2009, 'Low Reynolds number scalar transport enhancement in viscous and non-Newtonian fluid', International Journal of Heat and Mass Transfer, vol. 51, pp. 655-664.

Lester, DR, Buscall, R, Stickland, AD \& Scales, PJ 2014, 'Wall adhesion and constitutive modeling of strong colloidal gels', Journal of Rheology, vol. 58, pp. 1247-1276.

McFarlane, A, Yeap, KY, Bremmell, K \& Addai-Mensah, J 2008, 'The influence of flocculant adsorption kinetics on the dewaterability of kaolinite and smectite clay mineral dispersions', Colloids and Surfaces A - Physicochemical and Engineering Aspects, vol. 317, pp. 39-48.

Mizani, S, Soleimani, S \& Simms, P 2013, 'Effects of polymer dosage on dewaterability, rheology, and spreadability of polymeramended mature fine tailings', in RJ Jewell, AB Fourie, J Caldwell \& J Pimenta (eds), Proceedings of the 16th international seminar on paste and thickened tailings, InfoMine, Vancouver, pp. 117-131.

Mohanarangam, K, Yang, W \& Simic, K 2010, 'Application of ultrasonic velocity profiler (UVP) in the minerals industry', Proceedings of the $17^{\text {th }}$ Australasian fluid mechanics conference, University of Auckland, Auckland, paper 164. 
Mpofu, P, Addai-Mensah, J \& Ralston, J 2003, 'Investigation of the effect of polymer structure type on flocculation, rheology and dewatering behaviour of kaolinite dispersions', International Journal of Mineral Processing, vol. 71, pp. 247-268.

Mpofu, P, Addai-Mensah, J \& Ralston, J 2004, 'Flocculation and dewatering behaviour of smectite dispersions: effect of polymer structure type', Minerals Engineering, vol. 17, pp. 411-423.

Nguyen, TV, Farrow, JB, Smith J \& Fawell, PD 2012, 'Design and development of a novel thickener feedwell using computational fluid dynamics', Journal of The Southern African Institute of Mining and Metallurgy, vol. 112, pp. 939-948.

Ofori, P, Nguyen, AV, Firth, B, McNally, C \& Ozdemir, O 2011, 'Shear-induced floc structure changes for enhanced dewatering of coal preparation plant tailings', Chemical Engineering Journal, vol. 172, pp. 914-923.

Owen, AT, Fawell, PD, Swift, JD \& Farrow, JB 2002, 'The impact of polyacrylamide flocculant solution age on flocculation performance', International Journal of Mineral Processing, vol. 67, pp. 123-144.

Owen, AT, Fawell, PD \& Swift, JD 2007, 'The preparation and ageing of acrylamide/acrylate copolymer flocculant solutions', International Journal of Mineral Processing, vol. 84, pp. 3-14.

Peng, FF \& Di, P 1994, 'Effect of multivalent salts - calcium and aluminium on the flocculation of kaolin suspension with anionic polyacrylamide', Journal of Colloid and Interface Science, vol. 164, pp. 229-237.

Rey, PA 1988, 'The effect of water chemistry on the performance of anionic polyacrylamide-based flocculants', in BM Moudgil \& BJ Scheiner (eds), Flocculation and dewatering, Engineering Foundation, New York, pp. 195-214.

Rulyov, NN, Korolyov, BY \& Kovalchuk, NM 2006, 'Application of ultra-flocculation for improving fine coal concentrate dewatering', Coal preparation, vol. 26, no. 1, pp. 17-32.

Sabah, E \& Cengiz, I 2004, 'An evaluation procedure for flocculation of coal preparation plant tailings', Water Research, vol. 38, pp. 1542-1549.

Sabah, E \& Erkan, ZE 2006, 'Interaction mechanism of flocculants with coal waste slurry', Fuel, vol. 85, pp. 350-359.

Scott, JP, Fawell, PD, Ralph, DE \& Farrow, JB 1996, 'The shear degradation of high molecular weight flocculant solutions', Journal of Applied Polymer Science, vol. 62, pp. 2097-2106.

Tanguay, M, Fawell, P \& Adkins, S 2014, 'Modelling the impact of two different flocculants on the performance of a thickener feedwell', Applied Mathematical Modelling, vol. 38, pp. 4262-4276.

Witham, MI, Grabsch, AF, Owen, AT \& Fawell, PD 2012, 'The effect of cations on the activity of anionic polyacrylamide flocculant solutions', International Journal of Mineral Processing, vol. 114-117, pp. 51-62.

Yao, Y, van Tol, AF \& van Paassen, LA 2012, 'The effect of flocculant on the geotechnical properties of mature fine tailings: an experimental study, Proceedings of the 3rd international oil sands tailings conference, Edmonton, Alberta, pp. 391-398.

Zhu, Y, Wu, J, Shepherd, IS, Coghill, M, Vagias, N \& Elkin, K 2000, 'An automated measurement technique for slurry settling tests', Minerals Engineering, vol. 13, pp. 765-772. 
